\title{
Genetic dissection of upland cotton (Gossypium hirsutum) cultivars developed in Hubei Province by mapped SSRs
}

\author{
J.L. Tu ${ }^{1 *}$, M.J. Zhang ${ }^{2 *}$, X.Q. Wang ${ }^{1}$, X.L. Zhang ${ }^{1}$ and Z.X. Lin ${ }^{1}$ \\ ${ }^{1}$ National Key Laboratory of Crop Genetic Improvement, \\ National Centre of Plant Gene Research, Huazhong Agricultural University, \\ Wuhan, Hubei, China \\ ${ }^{2}$ Department of Bioscience and Engineering, Huanggang Normal College, \\ Huanggang, China \\ *These authors contributed equally to this study. \\ Corresponding author: Z.X. Lin \\ E-mail: linzhongxu@mail.hzau.edu.cn
}

Genet. Mol. Res. 13 (1): 782-790 (2014)

Received January 9, 2013

Accepted May 24, 2013

Published January 31, 2014

DOI http://dx.doi.org/10.4238/2014.January.31.4

\begin{abstract}
The genetic diversity of 51 upland cotton cultivars with different parental origins and breeding periods that were developed in Hubei Province was studied on the basis of 237 mapped simple sequence repeat markers covering the cotton genome. A total of 108 polymorphic primer pairs amplified 196 loci; the polymorphism information content range was 0.04 to 0.83 , with an average of 0.46 . A model-based clustering analysis (STRUCTURE) of the genomic data identified 3 clear subpopulations, and the result was confirmed by principal components analysis. The genetic similarity coefficient among 51 upland cotton cultivars was 0.598 on average, ranging from 0.378 to 0.817 . The unweighted pair group method with arithmetic average cluster analysis revealed inconsistencies in other clustering patterns: "Tianmian1" was distinct from the rest of the materials and formed a separate cluster. This study will provide a guide for breeders to develop
\end{abstract}


new cultivars efficiently and to choose parents, and it supports the need to introduce new alleles into the gene pool of the upland cotton breeding program in Hubei Province.

Key words: Upland cotton; Simple sequence repeats; Genetic diversity

\section{INTRODUCTION}

Cotton belongs to Dicotyledoneae, produces fiber from seed, and occupies a very important status in China's textile industry. Hubei Province, in the middle reaches of the Yangtze River, is one of the important cotton bases of China and makes a significant contribution to the textile industry. The cotton cultivars of Hubei Province mainly experienced 3 periods: varieties introduction, systematic selection, and cross breeding. Before the 1960s, upland cotton varieties that were introduced from America were mainly cultivated, especially Deltapine 15. In the 1960s and 1970s, Eguangmian was mainly cultivated by Chinese cotton breeders. Subsequently, cotton cultivars with high-yield production and hybrid varieties were introduced. In the 21 st century, high-yield, disease-resistant, and insect-resistant hybrid cultivars were mainly cultivated.

Molecular markers that detect the organ's genetic diversity effectively should have the following characteristics: rich polymorphism, stability, and high heredity. DNA molecular markers were the most effective method of genetic analysis at the DNA level. In cotton, various molecular markers have been applied in genetic diversity analysis such as simple sequence repeats (SSRs), rapid amplification of polymorphic DNA (RAPD), amplified fragment length polymorphism, inter-SSR, and sequence-related amplified polymorphism; the cotton species included Gossypium hirsutum (Xu et al., 2001; Rana et al., 2005; Zhang et al., 2005; Wu et al., 2010; Kalivas et al., 2011), G. barbadense (Westengen et al., 2005; El-Zanaty et al., 2011; Wang et al., 2011), and G. arboretum (Liu et al., 2006; Kantartzi et al., 2009).

An understanding of the magnitude and patterns of genetic diversity in upland cotton has important implications for breeding programs and for the conservation of genetic resources. $\mathrm{Lu}$ and Myers (2002) used RAPD data to evaluate genetic relationships and discriminate 10 influential upland cotton varieties (G. hirsutum). Chen and Du (2006) described the genetic diversity of 43 sources of upland cotton in China on the basis of SSR markers, and the results showed that the early maturity cotton accessions in the north were higher than those from the Huanghe and Yangtze growing areas; also, the molecular marker genetic similarity index of the domestic varieties was higher than that of the imported varieties. Kalivas et al. (2011) used SSR markers for the identification and the phylogenetic analysis of 29 cultivars of G. hirsutum. The polymorphism information content (PIC) ranged from 0 to 0.548 with a mean of 0.293 ; the unweighted pair group method with arithmetic average (UPGMA) analysis showed that the 30 genotypes formed 3 main groups. Zhang et al. (2011) estimated the genetic diversity of 59 core cotton cultivars from China's main cotton-growing areas based on genomic and newly developed expressed sequence tag (EST)-SSR markers.

In this study, 51 upland cotton cultivars that were developed from different periods and sources, according to the development of cotton breeding history of Hubei Province, were selected to analyze and evaluate their genetic relationship on the basis of mapped SSR markers. Combining 3 diversity analysis methods, 51 upland cotton cultivars could be deeply and objectively investigated. 


\section{MATERIAL AND METHODS}

\section{Plant materials and DNA extraction}

Fifty-one upland cotton cultivars that were developed in Hubei Province were used in this study (Table 1). Cotton genomic DNA was extracted from young leaves with the modified cetyltrimethylammonium bromide (CTAB) method (Paterson et al., 1993).

Table 1. Name, origin, and breeding period of upland cotton cultivars developed in Hubei Province.

\begin{tabular}{|c|c|c|c|c|}
\hline Cultivar & Code & Female parent & Male parent & Developed year \\
\hline Deltapine15 & HB01 & & & 1947 \\
\hline Yapengmian & HB02 & Deltapine15 mutant & & 1956 \\
\hline Dongting 1 & HB03 & Deltapine15 Breeding & & 1957 \\
\hline Eguangmian & HB04 & Eguangmian & & 1964 \\
\hline Emian 1 & HB05 & Deltapine15 Breeding & & $1950 \mathrm{~s}$ \\
\hline Emian 2 & HB06 & Deltapine 15 x Yapengmian & & 1959 \\
\hline Emian 3 & HB07 & Deltapine15 Breeding & & $1950 \mathrm{~s}$ \\
\hline Emian 4 & HB08 & Deltapine15 Breeding & & 1962 \\
\hline Emian 5 & HB09 & Deltapine15 Breeding & & $1960 \mathrm{~s}$ \\
\hline Emian 6 & HB10 & Deltapine 15 mutant & & 1966 \\
\hline Emian 9 & HB11 & Deltapine 15 mutant & & 1964 \\
\hline Emian 10 & $\mathrm{HB} 12$ & Deltapine15 Breeding & & 1968 \\
\hline Emian11 & HB13 & Dai3599 x Gaomi933 & & 1980 \\
\hline Emian 12 & HB14 & {$[(5006 \times 1080)(3762 \times$ Mujin $)] F_{1}$} & Henan 79 & 1985 \\
\hline Emian 13 & HB15 & CRI10 & & 1990 \\
\hline Emian 14 & HB16 & 2930 & CRI7 & 1988 \\
\hline Emian 15 & HB17 & $60 \mathrm{Co}$ radiation & & 1988 \\
\hline Emian 16 & HB18 & Ejing1 & Esha28 & 1990 \\
\hline Emian 17 & HB19 & & & 1988 \\
\hline Emian 18 & HB20 & Wan73-10 & Shahu49 & 1993 \\
\hline Emian 19 & $\mathrm{HB} 21$ & Eing $1 \times$ Zhongmianxin 10 & & 1991 \\
\hline Emian 20 & $\mathrm{HB} 22$ & Ejing $1 \times$ Xiangmian 10 & & 1992 \\
\hline Emian 21 & $\mathrm{HB} 23$ & $\left(5512 \times\right.$ PD4548)F $\mathrm{F}_{1} \times \mathrm{CRI} 12$ & & 1994 \\
\hline Emian 23 & $\mathrm{HB} 24$ & $98-38 \times 4643 \times 3247$ & & 2003 \\
\hline Emian 24 & $\mathrm{HB} 25$ & Simian3 & $87-28$ & 2004 \\
\hline Esha28 & HB26 & Jingmian x Gangmian1 & Jingmian 4 & 1981 \\
\hline Ejing 1 & HB27 & $(3208 \times$ Jingmian 4$) \mathrm{F}_{2}$ & AntongSP21 & 1985 \\
\hline Ejing 92 & HB28 & 3208 & Jingmian4 & 1983 \\
\hline Ekangmian1 & HB29 & Jing 3247 x Jiangsu9118 & & 1990 \\
\hline Ekangmian 2 & HB30 & Ejing1 x Zhong2535 & & 1998 \\
\hline Ekangmian 3 & HB31 & Eguangmian x Jingzhou 3247 & Zhong2535 & 1995 \\
\hline Ekangmian 4 & HB32 & Ejing1 & & 1996 \\
\hline Ekangmian 5 & HB33 & Emian 12 x PD0113 & & 1992 \\
\hline Ekangmian 6 & HB34 & Ejing 1 & Xiangmian 10 & 1997 \\
\hline Ekangmian 7 & HB35 & Jing7701 & Jing 1418 & 1997 \\
\hline Ekangmian 8 & HB36 & $\left(3247 \times\right.$ Esha28) $F_{1} \times$ CCRI12 & & 1998 \\
\hline Ekangmian 9 & HB37 & Ejing 1 & Zhong7263+M03 & 1999 \\
\hline Ekangmian 10 & HB38 & (Jing3117 x Zhong12)F $\mathrm{F}_{1}$ x Jing3187 & & 1999 \\
\hline Tianmian1 & HB39 & Humian204 & & 1969 \\
\hline Shanong6 & HB40 & Eguangmian & & 1965 \\
\hline Deltapine16 & HB41 & Eguangmian & Deltapine45 & 1970 \\
\hline Gangmian1 & HB42 & Eguangmian & & 1972 \\
\hline Gangmian25 & HB43 & Gangmian1 & & 1978 \\
\hline Ekangchong1 & HB44 & Simian3 import GFMcryIABt & & 2002 \\
\hline Yishuhong & HB45 & Tuozimian & & 1953 \\
\hline Bayimian & HB46 & Shuangtaomian $\mathrm{x}$ Eguangmian & & 1970 \\
\hline Xiaomian1 & HB47 & Eguangmian & & \\
\hline Jing 1246 & HB48 & Ejing1 & Xiangmian10 & 1997 \\
\hline Jingchu201 & HB49 & Ekangmian 10 & 3259 & 2005 \\
\hline Huakangmian 1 & HB50 & 168 & Sumian3 & 2002 \\
\hline Emian 22 & HB51 & Ejing1 x PD2165 & & 1994 \\
\hline
\end{tabular}




\section{SSR marker analysis}

SSR markers were the 237 pairs of SSRs that were selected from 26 chromosomes and were included 4 to 17 markers per chromosome (Wang et al., 2011). Polymerase chain reaction (PCR), electrophoresis and silver staining were performed as previously described by Wang et al. (2011).

\section{Data analysis}

After observing the PCR electrophoresis results, the bands of DNA fragments were scored " 1 " for presence, "0" for absence, and " 9 " for missing data. Genetic diversity analysis and further study were made on the basis of these scores. The statistical methods and formulae that were used are shown below.

1) $\mathrm{PIC}=1-\Sigma \mathrm{P} i^{2}(i=1-\mathrm{K})$, where $P i$ represents the variation in frequency of the $i$ th allele, and $K$ represents the sum allele of one marker.

2) The STRUCTURE version 2.1 (Pritchard et al., 2000) software was used to analyze the population structure of these accessions. Markov Chain Monte Carlo was set to 50,000, burn-in was set to 100,000 , and the $\mathrm{K}$ value was from 2 to 10 and each was repeated 3 times.

3) The genetic diversity statistics of upland cotton were analyzed using the frequency and analysis of molecular variance (AMOVA) functions of the GenAlEx6.2 software (Cruz et al., 2007), including polymorphism percentage, number of effective alleles, Shannon's information index, genetic differentiation (PhiPT), gene flow, and expected heterozygosity.

4) AMOVA was used to estimate the molecular diversity between and within populations, and principal coordinates analysis (PCA) based on Nei's genetic distance was used to confirm the Structure results.

5) Genetic similarity coefficients (Jaccard's coefficients) among varieties were calculated using the Qualitative Date program of the NTSYSpc 2.1 software (Biostatistics Inc., New York, USA). The dendrograms were constructed by UPGMA.

\section{RESULTS}

\section{Amplified efficiency of SSRs}

Among the 237 SSR primer pairs, 108 primer pairs (45.6\%) were polymorphic and generated 196 polymorphic loci with an average of 1.82 loci per primer pair. Among them, 51 pairs of primers amplified only 1 polymorphic locus, 33 amplified 2 loci, 17 amplified 3 loci, and 7 pairs amplified 4 polymorphic loci. The PIC value for the SSR loci ranged from 0.04 to 0.83 with a mean of 0.46 . The SSR with the highest average PIC value was BNL3257. When taking chromosomes into account, the highest average PIC values were for Chr17 (0.71), Chr04 (0.69), and Chr24 (0.66) (Table 2).

\section{Population structure analysis}

STRUCTURE V2.31 was used to infer the population structure using the entire SSR marker dataset. With $\mathrm{K}$ running, the distribution of $\mathrm{L}(\mathrm{K})$ did not show a clear mode for the 
true $\mathrm{K}$ (Figure 1A). Thus, another method was used to overcome the difficulty of interpreting the real $\mathrm{K}$ values (Evanno et al., 2005), $\Delta \mathrm{K}=\mathrm{m}(|\mathrm{L}(\mathrm{K}+1)-2 \mathrm{~L}(\mathrm{~K})+\mathrm{L}(\mathrm{K}-1)|) / \mathrm{s}[\mathrm{L}(\mathrm{K})]$, where $\Delta \mathrm{K}$ was developed and showed a clear peak where the true value of $\mathrm{K}$ was present. In this study, the highest value of $\Delta K$ for the 51 cultivars was $K=3$ (Figure $1 B$ ), so value of $K=$ 3 was chosen for the analysis. As shown in Figure 2, the cultivars were clearly classified into 1 of the 3 subpopulations (P1-P3), which included 7, 29, and 15 cultivars, respectively (Table 3).

Table 2. Amplified efficiency of markers on different chromosomes.

\begin{tabular}{|c|c|c|c|c|}
\hline Chromosome & Number of markers & Amplified loci & PIC value & Mean PIC value \\
\hline$\overline{C h r 1}$ & 6 & 4 & $0.04-0.66$ & 0.35 \\
\hline $\mathrm{Chr} 2$ & 5 & 4 & $0.04-0.72$ & 0.38 \\
\hline Chr3 & 7 & 5 & $0.40-0.72$ & 0.61 \\
\hline Chr4 & 5 & 8 & $0.61-0.78$ & 0.69 \\
\hline Chr5 & 11 & 4 & $0.04-0.44$ & 0.14 \\
\hline Chr6 & 8 & 4 & $0.36-0.72$ & 0.54 \\
\hline Chr7 & 4 & 1 & 0.29 & 0.29 \\
\hline Chr8 & 12 & 12 & $0.08-0.83$ & 0.45 \\
\hline Chr9 & 11 & 12 & $0.26-0.82$ & 0.63 \\
\hline Chr10 & 9 & 7 & $0.04-0.71$ & 0.43 \\
\hline Chr11 & 17 & 10 & $0.04-0.82$ & 0.27 \\
\hline Chr12 & 14 & 14 & $0.04-0.79$ & 0.50 \\
\hline Chr13 & 11 & 13 & $0.08-0.8$ & 0.51 \\
\hline Chr14 & 6 & 7 & $0.04-0.75$ & 0.38 \\
\hline Chr15 & 11 & 6 & $0.11-0.72$ & 0.46 \\
\hline Chr16 & 6 & 7 & $0.04-0.71$ & 0.50 \\
\hline Chr17 & 5 & 3 & $0.70-0.72$ & 0.71 \\
\hline Chr18 & 7 & 10 & $0.04-0.82$ & 0.51 \\
\hline Chr19 & 13 & 8 & $0.11-0.75$ & 0.45 \\
\hline Chr20 & 13 & 9 & $0.11-0.74$ & 0.49 \\
\hline Chr21 & 6 & 10 & $0.08-0.80$ & 0.61 \\
\hline Chr23 & 9 & 11 & $0.08-0.80$ & 0.53 \\
\hline Chr24 & 11 & 5 & $0.53-0.78$ & 0.66 \\
\hline Chr25 & 17 & 10 & $0.04-0.61$ & 0.31 \\
\hline Chr26 & 13 & 12 & $0.14-0.78$ & 0.50 \\
\hline
\end{tabular}

$\mathrm{PIC}=$ polymorphism information content.
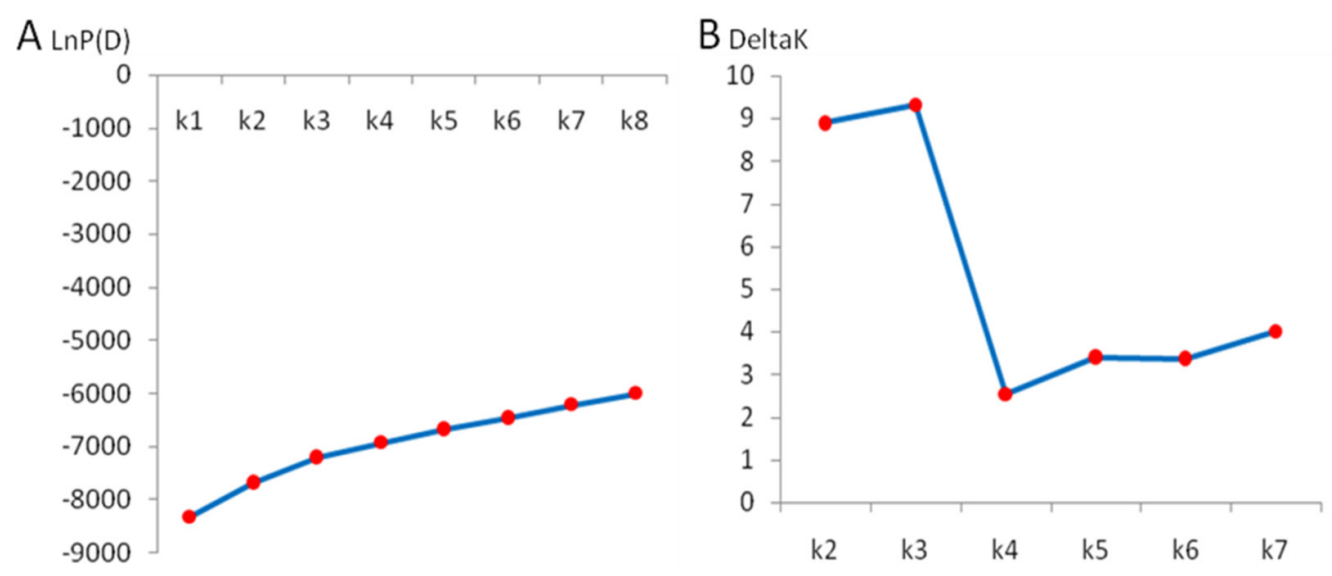

Figure 1. Two different methods of determining the value of K. A. The ad hoc procedure described by Pritchard et al. (2000). B. The second order statistics $(\Delta K)$ developed by Evanno et al. (2005). 

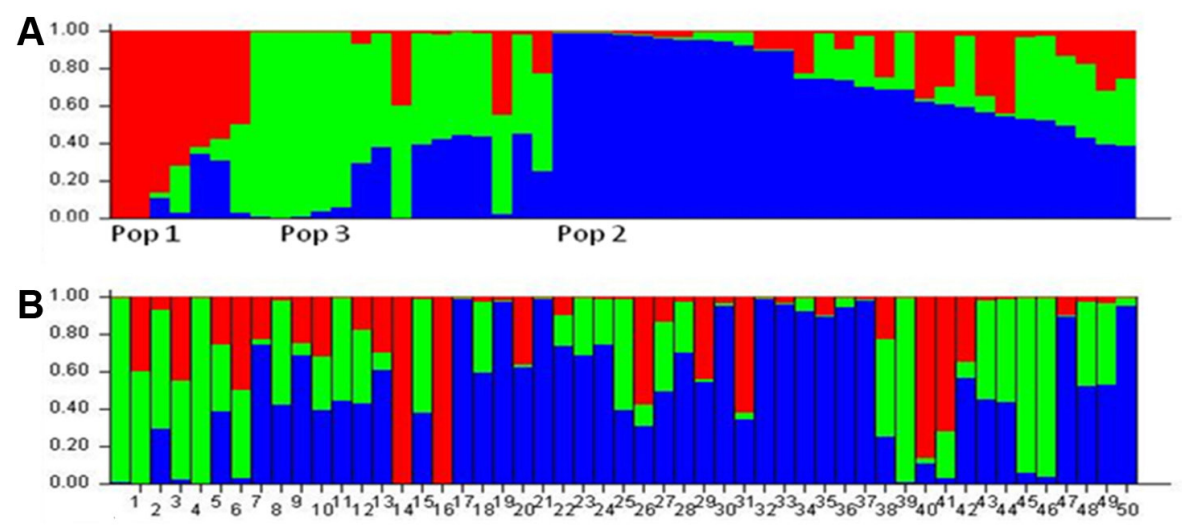

Figure 2. A. Model-based ancestry for each of the 51 upland cotton cultivars based on SSR markers used to build the Q matrix. Red represents Pop 1 and blue represents Pop 2 while green is representative of Pop 3. B. Population structure of 51 upland cotton cultivars; the height of vertical axis means that the material is divided into a special cluster where the similarity of each line is more than $50 \%$.

Table 3. Cultivars' distribution and names of STRUCTURE-based populations.

\begin{tabular}{ll}
\hline Group & Cultivar No. \\
\hline Pop 1 (7) & HB07 HB15 HB17 HB27 HB32 HB41 HB42 \\
Pop 2 (29) & HB06 HB08 HB10 HB11 HB13 HB14 HB18 HB19 HB20 HB21 HB22 HB23 HB24 HB25 HB28 \\
& HB29 HB30 HB31 HB33 HB34 HB35 HB36 HB37 HB38 HB43 HB48 HB49 HB50 HB51 \\
Pop 3 (15) & HB01 HB02 HB03 HB04 HB05 HB09 HB12 HB16 HB26 HB39 HB40 HB44 HB45 HB46 HB47 \\
\hline
\end{tabular}

\section{PCA and AMOVA results}

PCA was conducted to further assess the population subdivisions that were identified by STRUCTURE. In the PCA graph (Figure 3), principal coordinates 1, 2, and 3 explained $27.52,19.12$, and $14.97 \%$ of variation, respectively. The PCA results absolutely confirmed the results that were obtained from STRUCTURE, and genetic diversity statistics showed that the order of all statistical values was P2 $>$ P3 $>$ P1. Moreover, the 3 populations had different statistical values in pairwise comparisons (Table 4).

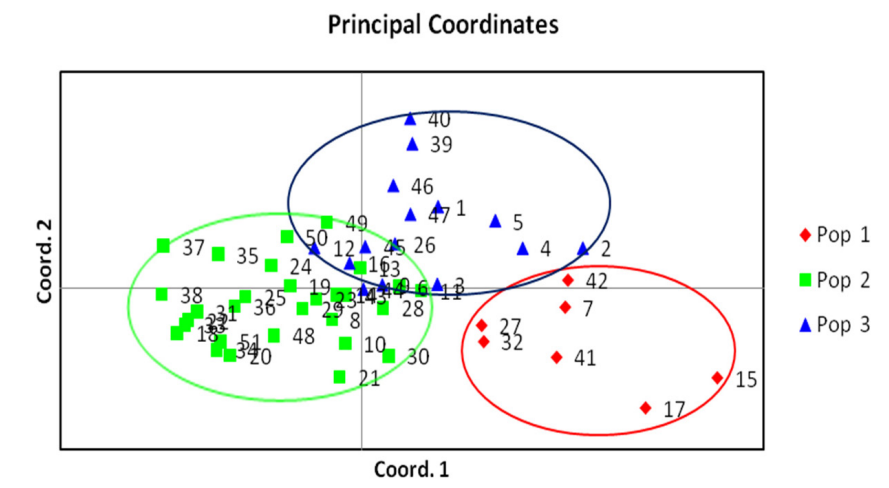

Figure 3. PCA graph of 51 upland cotton cultivars developed in Hubei Province. 
Table 4. Comparison of genetic diversity among subgroups.

\begin{tabular}{lccccccc}
\hline Population & $\mathrm{N}$ & $N_{\mathrm{A}}$ & $N_{\mathrm{E}}$ & $\mathrm{I}$ & $H_{\mathrm{E}}$ & $\mathrm{U} H_{\mathrm{E}}$ & Polymorphism (\%) \\
\hline Pop 1 & 7 & 1.347 & 1.317 & 0.285 & 0.188 & 0.203 & 57.28 \\
Pop 2 & 29 & 1.751 & 1.383 & 0.351 & 0.229 & 0.233 & 84.51 \\
Pop 3 & 15 & 1.512 & 1.362 & 0.325 & 0.214 & 0.221 & 71.36 \\
Total & 51 & 1.537 & 1.354 & 0.321 & 0.210 & 0.219 & 71.05 \\
\hline
\end{tabular}

$N_{\mathrm{A}}=$ number of alleles; $N_{\mathrm{E}}=$ effective number of alleles; I $=$ Shannon's information index; $H_{\mathrm{E}}=$ expected heterozygosity; $\mathrm{U} H_{\mathrm{E}}=$ unbiased $H_{\mathrm{E}}$.

According to the Structure result, the 51 cultivars were divided into 3 subgroups. AMOVA showed that the between-population component of genetic variance was $15 \%$, in contrast to $85 \%$ for the within-population component (Table 5). At P $<0.01$, the general PhiPT was 0.146 .

Table 5. Analysis of molecular variance.
\begin{tabular}{lccccc}
\hline Source & d.f. & SS & MS & Estimated variation & $\%$ \\
\hline Among population & 2 & 172.36 & 86.18 & 4.22 & $15 \%$ \\
Within population & 48 & 1184.11 & 24.67 & 24.67 & $85 \%$ \\
Total & 50 & 1356.47 & & 28.89 & $100 \%$ \\
\hline
\end{tabular}

d.f. = degrees of freedom; $\mathrm{SS}=$ sum of squares; MS = mean squares.

\section{UPGMA analysis}

Genetic similarity coefficient matrices were calculated by the NTSYS-pc 2.1 software package (Rohlf, 2000) based on Jaccard's coefficient. The resulting similarity coefficient was used to construct a genetic distance matrix using the SAHN procedure based on UPGMA (Figure 4). The genetic distance between these selected cultivars ranged from 0.378 to 0.817 with an average of 0.598 , and the highest value that were observed was between HB01 and HB47, which were both introduced from America in the 1960s. According to the UPGMA dendrogram, HB39 was distinct from the rest of materials and formed a separate cluster. The rest of the cultivars could be separated into 2 clusters. HB02, HB04, HB15, HB17, HB27, HB41, and HB42 were grouped together to form the second cluster, and the others formed the third cluster.

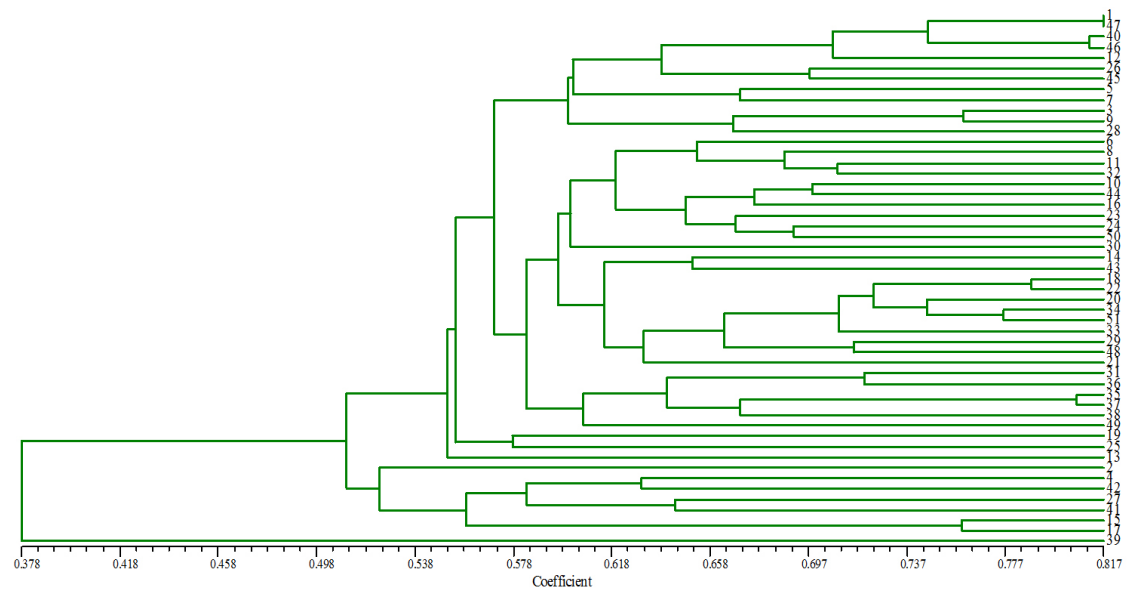

Figure 4. UPGMA cluster analysis of 51 upland cotton cultivars based on SSR markers. 


\section{DISCUSSION}

To evaluate the overall genetic variation among different cotton populations, the genetic diversity of 51 upland cotton cultivars that were developed in different periods in Hubei Province were analyzed. The range of PIC calculated in this study was $0.04-0.83$ with an average of 0.46 . The average genetic similarity coefficient of 51 cultivars was 0.598 and ranged from 0.378 to 0.817 . This result indicates that the genetic variation among those cultivars is very low, which is consistent with previous studies (Wendel et al., 1992; Iqbal et al., 1997, 2001; $\mathrm{Xu}$ et al., 2001). These indicate that the breeding of upland cotton faces a bottleneck effect. Therefore, introduction of new cultivars and new breeding methods is urgent and necessary. The comparison of the genetic diversity of 3 subpopulations derived from the analysis of population structure implicated that the genetic variation among these 3 subpopulations was very small.

AMOVA showed that the between-population component of genetic variance was $15 \%$, and the within-population component was $85 \%$. That is, the variation among different cotton sources mainly contributed to the overall variation. At $\mathrm{P}<0.01$, the general PhiPT was 0.145 , which indicated that the molecular variation was mostly restricted to the subpopulations. Identifying a proper $\mathrm{K}$ value to divide the population structure is a prerequisite to assess the genetic variation and structure at the molecular level using the computational program STRUCTURE (Zhao et al. 2010). It was very difficult to define the $\mathrm{K}$ value based on the LnP (D) value with slow increasing tendency. Therefore, the peak value could be easily observed when the $\mathrm{K}$ value was set to 3 based on the $\Delta \mathrm{K}$ variation that was calculated using the reported method (Evanno et al., 2005). Besides Structure, UPGMA and PCA were used to divide the population structure (Shim and Jørgensen, 2000; Li et al., 2010; Sakiroglu et al., 2010). In our study, subpopulations were categorized by 3 different methods. According to our results, Structure and PCA analysis were highly consistent. Comparatively, the results consistently showed that HB15, HB17, HB27, HB41, and HB42 were grouped together by Structure, PCA, and UPGMA; on the other hand, the results of UPGMA showed some difference from the other 2 methods because the different methods analyzed genetic diversity according to different theories, resulting in different results among them.

In summary, systematic evaluation of the genetic diversity of upland cotton cultivars in Hubei Province has been carried out in this study. This study will provide very useful information for future cotton breeding in Hubei Province. This research also provides a very strong theoretical basis to obtain an excellent and scientifically established repository of the Hubei cotton varieties.

\section{ACKNOWLEDGMENTS}

Research supported by the National Basic Research Program (\#2011CB109303) and the Fundamental Research Funds for the Central Universities (Program \#2012MBDX007).

\section{REFERENCES}

Chen G and Du XM (2006). Genetic diversity of source germplasm of Upland cotton in China as determined by SSR marker analysis. Yi. Chuan Xue. Bao. 33: 733-745.

Cruz VMV, Luhman R, Marek LF, Rife CL, et al. (2007). Characterization of flowering time and SSR marker analysis of spring and winter type Brassica napus L. germplasm. Euphytica 153: 43-57. 
El-Zanaty AM, Salem KFM and Esmail RM (2011). Detection of genetic diversity in Egyptian cotton (Gossypium barbadense L.) varieties using RAPD markers and morphological traits. J. Am. Sci. 7: 1107-1115.

Evanno G, Regnaut S and Goudet J (2005). Detecting the number of clusters of individuals using the software STRUCTURE: a simulation study. Mol. Ecol. 14: 2611-2620.

Iqbal MJ, Aziz N, Saeed NA, Zafar Y, et al. (1997). Genetic diversity evaluation of some elite cotton varieties by RAPD analysis. Theor. Appl. Genet. 94: 139-144.

Iqbal MJ, Reddy OUK, El-Zik KM and Pepper AE (2001). A genetic bottleneck in the 'evolution under domestication' of upland cotton Gossypium hirsutum L. examined using DNA fingerprinting. Theor. Appl. Genet. 103: 547-554.

Kalivas A, Xanthopoulos F, Kehagia O and Tsaftaris AS (2011). Agronomic characterization, genetic diversity and association analysis of cotton cultivars using simple sequence repeat molecular markers. Genet. Mol. Res. 10: 208-217.

Kantartzi SK, Ulloa M, Sacks E and Stewart JM (2009). Assessing genetic diversity in Gossypium arboreum L. cultivars using genomic and EST-derived microsatellites. Genetica 136: 141-147.

Li JQ, Schulz B and Stich B (2010). Population structure and genetic diversity in elite sugar beet germplasm investigated with SSR markers. Euphytica 175: 35-42.

Liu DQ, Guo XP, Lin ZX, Nie YC, et al. (2006). Genetic diversity of Asian cotton (Gossypium arboreum L.) in China evaluated by microsatellite analysis. Genet. Resour. Crop Evol. 53: 1145-1152.

$\mathrm{Lu} \mathrm{J}$ and Myers O (2002). Genetic relationships and discrimination of ten influential Upland cotton varieties using RAPD markers. Theor. Appl. Genet. 105: 325-331.

Paterson AH, Brubaker CL and Wendel JF (1993). A rapid method for extraction of cotton (Gossypium spp.) genomic DNA suitable for RFLP or PCR analysis. Plant Mol. Biol. Rep. 11: 122-127.

Pritchard JK, Stephens M and Donnelly P (2000). Inference of population structure using multilocus genotype data. Genetics 155: 945-959.

Rana MK, Singh VP and Bhat KV (2005). Assessment of genetic diversity in upland cotton (Gossypium hirsutum L.) breeding lines by using amplified fragment length polymorphism (AFLP) markers and morphological characteristics. Genet. Resour. Crop Evol. 52: 989-997.

Rohlf FJ (2000). NTSYS-pc: Numerical Taxonomy and Multivariate Analysis System, Version 2.1. Exeter Software, New York

Sakiroglu M, Doyle JJ and Charles BE (2010). Inferring population structure and genetic diversity of broad range of wild diploid alfalfa (Medicago sativa L.) accessions using SSR markers. Theor. Appl. Genet. 121: 403-415.

Shim SI and Jørgensen RB (2000). Genetic structure in cultivated and wild carrots (Daucus carota L.) revealed by AFLP analysis. Theor. Appl. Genet. 101: 227-233.

Wang XQ, Feng CH, Lin ZX and Zhang XL (2011). Genetic diversity of sea-island cotton (Gossypium barbadense) revealed by mapped SSRs. Genet. Mol. Res. 10: 3620-3631.

Wendel JF, Brubaker CL and Percival AE (1992). Genetic diversity in Gossypium hirsutum and the origin of upland cotton. Am. J. Bot. 79: 1291-1310.

Westengen OT, Huaman Z and Heun M (2005). Genetic diversity and geographic pattern in early South American cotton domestication. Theor. Appl. Genet. 110: 392-402.

Wu DP, Fang XX, Ma MN, Chen JH, et al. (2010). Genetic relationship and diversity of the germplasms in Gossypium barbadense L. from four different countries using SSR markers. Cotton Sci. 22: 104-109.

Xu QH, Zhang XL and Feng CD (2001). Genetic diversity analysis on cultivars (G. hirsutum L.) developed by Hebei Province and CCRI by RAPD markers. Acta Gossypii Sin. 13: 238-242.

Zhang JF, Lu Y, Cantrell RG and Hughs E (2005). Molecular marker diversity and field performance in commercial cotton cultivars evaluated in the Southwestern USA. Crop Sci. 45: 1483-1490.

Zhang Y, Wang XF, Li ZK, Zhang GY, et al. (2011). Assessing genetic diversity of cotton cultivars using genomic and newly developed expressed sequence tag-derived microsatellite markers. Genet. Mol. Res. 10: 1462-1470.

Zhao WG, Chung JW, Cho YI, Rha WH, et al. (2010). Molecular genetic diversity and population structure in Lycium accessions using SSR markers. C. R. Biol. 333: 793-800. 